hep-th/9811028

CALT-68-2199

\title{
On QCD String Theory and AdS Dynamics
}

\author{
Petr Hořava \\ California Institute of Technology, Pasadena, CA 91125, USA \\ horava@theory. caltech.edu \\ and \\ Department of Physics and Astronomy, Rutgers University \\ Piscataway, NJ 08855, USA
}

\begin{abstract}
The AdS/CFT correspondence of elementary string theory has been recently suggested as a "microscopic" approach to QCD string theory in various dimensions. We use the microscopic theory to show that the ultraviolet regime on the string world-sheet is mapped to the ultraviolet effects in QCD. In the case of $\mathrm{QCD}_{2}$, a world-sheet path integral representation of QCD strings is known, in terms of a topological rigid string theory whose world-sheet supersymmetry is reminiscent of Parisi-Sourlas supersymmetry. We conjecture that the supersymmetric rigid string theory is dual to the elementary Type IIB string theory in the singular AdS background that corresponds to the large- $N$ limit of $\mathrm{QCD}_{2}$. We also generalize the rigid string with world-sheet Parisi-Sourlas supersymmetry to dimensions greater than two, and argue that the theory is asymptotically free, a nonzero string tension is generated dynamically through dimensional transmutation, and the theory is topological only asymptotically in the ultraviolet.
\end{abstract}

October 1998 


\section{Introduction}

The AdS/CFT correspondence [1] 3] relates quantum gravity in AdS spaces to superconformal field theories on the boundary of the AdS. This intriguing relation between gravity and field theory has been much studied recently, and used as a new method for understanding strongly coupled gauge dynamics in various dimensions. Among the cases with maximum supersymmetry are [1] the duality between $\mathcal{N}=4 S U(N)$ super Yang-Mills theory in four dimensions and Type IIB theory on $\mathrm{AdS}_{5} \times \mathbf{S}^{5}$ with radius related to the 't Hooft coupling via $\left(g_{4}^{2} N\right)^{1 / 4}$, and with $N$ units of RR flux through $\mathbf{S}^{5}$; and the duality between M-theory on $\mathrm{AdS}_{7} \times \mathbf{S}^{4}$ and the $(0,2)$ theory in six dimensions.

In [2, [4] this correspondence was further extended to supersymmetric theories at finite temperature and to non-supersymmetric theories. In these cases, the dominant configuration on the AdS side is typically an AdS black hole with appropriate boundary conditions. Thus, non-supersymmetric $S U(N)$ Yang-Mills theory in four dimensions (pure $\mathrm{QCD}_{4}$ ) has been constructed by compactifying the six-dimensional $(0,2)$ theory to four dimensions on a torus with particular supersymmetry breaking periodicity conditions. On the AdS side, the limit that correspond to pure $\mathrm{QCD}_{4}$ leaves one dimension asymptotic to $\mathbf{S}^{1}$ very small, and the M-theory background becomes a certain limit of the AdS Schwarzschild black hole in Type IIA string theory.

These results provide some new support to the old suspicion that the master field of $\mathrm{QCD}_{4}$ may come in the form of a string theory 1 However, the regime in which supergravity calculations are reliable corresponds to large values $\lambda \gg 1$ of the 't Hooft coupling $\lambda=g_{4}^{2} N$, which translates on the gauge theory side to the strongly coupled gauge theory with a fixed UV cutoff $\Lambda$ set by the radius of the supersymmetry-breaking dimension [7]. The continuum limit of $\mathrm{QCD}_{4}$ lies in the regime where $\lambda$ is sent to zero as $\Lambda$ goes to infinity,

$$
\lambda \sim \frac{\beta_{0}}{\ln \left(\Lambda / \Lambda_{\mathrm{QCD}}\right)},
$$

in accord with asymptotic freedom. This limit is clearly difficult to understand on the string theory side, since it corresponds to Type IIA string theory in a rather singular limit of the corresponding AdS black hole background. Moreover, it seems rather likely that the regimes with small and large $\lambda$ are separated by a phase transition [7-9].

Thus, the construction of the master field of pure $\mathrm{QCD}_{4}$ in the continuum limit relies, at least in this approach, on our ability to understand the $\lambda \rightarrow 0$ limit (1.1) on

1 For some background on the idea of QCD strings, see e.g. [5, 6]. 
the string theory side of the correspondence. The AdS construction indicates that QCD glueballs should correspond on the Type IIA string theory side to supergravitons [7] in the background of the AdS black hole. In the $\lambda \rightarrow 0$ limit of continuum $\mathrm{QCD}_{4}$, we expect that the lowest closed string state should develop a non-zero mass gap of order $\Lambda_{\mathrm{QCD}}$. Any direct understanding of such "dressing" of the supergraviton seems very difficult in the elementary string theory approach, in particular if we try to extrapolate from the $\lambda \gg 1$ regime of small curvature.

The AdS/CFT correspondence may provide a microscopic theory of the QCD master field in principle, in terms of the corresponding limit of the Type IIA string background. One can certainly hope that a detailed understanding of the Type IIA string theory sigma model in the RR background and in the singular, large curvature limit will give satisfactory answers to some of these puzzling questions. However, to understand the continuum limit of $\mathrm{QCD}_{4}$ using the AdS construction, we do not need to understand Type IIA string theory on this class of backgrounds; this class contains non-universal information which will be irrelevant in the continuum $\mathrm{QCD}_{4}$ limit. For example, in the continuum limit we expect various states of the full elementary string theory - in particular, the $S O(6)$ non-singlet Kaluza-Klein modes on $\mathbf{S}^{5}$ - to decouple from the part of the spectrum corresponding to QCD.

If we are only interested in the universal properties of continuum QCD, and given the obvious difficulty with understanding the full Type IIA string theory on this class of backgrounds, it may be natural to search for alternatives, in the form of an effective string theory that would directly describe the rather difficult singular limit of Type IIA string theory. The purpose of this paper is to initiate some steps in such an effective string theory approach.

In Section 2 we present some general discussion of scales in the hypothetical QCD string theory, and use the AdS/CFT relation to derive a "UV-UV correspondence" between the string world-sheet and the space-time of QCD.

In Section 3, we discuss the case of pure $\mathrm{QCD}_{2}$ at large- $N$. In this particular case, a string theory whose path integral reproduces the large- $N$ expansion of $\mathrm{QCD}_{2}$ is known 10 [12]. This theory was originally constructed as a topological rigid string theory, but we will see later in the paper that it is more natural to think of the theory of [10,12] as a rigid string theory with world-sheet Parisi-Sourlas supersymmetry. We review some of the properties of this two-dimensional rigid string theory as a theory of $\mathrm{QCD}_{2}$ strings in Section 3.1. In 
Section 3.2, using the AdS/CFT technology, we construct pure $\mathrm{QCD}_{2}$ by compactifying $\mathcal{N}=4$ super Yang-Mills theory from four dimensions. In the corresponding limit of pure $\mathrm{QCD}_{2}$ at large $N$ and fixed but small 't Hooft coupling, the theory should be described by Type IIB string theory on an asymptotically AdS background in a rather singular limit. In Section 3.3 we compare the two approaches, and conjecture that Type IIB string theory in the singular limit of the AdS background is equivalent to the supersymmetric rigid string of Section 3.1.

Given this relation between the supersymmetric rigid string theory on one hand, and the Type IIB string theory in the singular background corresponding to $\mathrm{QCD}_{2}$ on the other, we study possible extensions of the supersymmetric rigid string theory to higher dimensions. In Section 4, we find a very natural generalization of the supersymmetric rigid string of Section 3.1 to dimensions greater than two, in the form of a rigid string theory with local Parisi-Sourlas supersymmetry. Symmetries of this theory do not allow any tension term in the Lagrangian, and the theory has only one marginal coupling - the string rigidity $\alpha_{0}$. We calculate the one-loop beta function for $\alpha_{0}$, and find that the theory is asymptotically free in dimensions greater than two. We argue that due to dimensional transmutation on the world-sheet, a non-zero string tension is dynamically generated at large distances, and supersymmetry is broken. In contrast, the two-dimensional theory is supersymmetric at all scales, and the Parisi-Sourlas supersymmetry effectively plays the role of topological BRST invariance. In dimensions higher than two, the string has physical oscillations due to non-zero tension at large distances, but becomes asymptotically topological due to the restoration of supersymmetry in the UV.

Even though the main motivation for our study of the supersymmetric rigid string in dimensions higher than two originates largely from the arguments presented earlier in Sections $1-3$ of the paper, Section 4 can be read essentially independently of the rest of the paper.

\section{Correspondence of Scales in QCD String Theory}

The AdS/CFT correspondence relates a theory with gravity in the bulk to a gauge theory at the boundary. As was pointed out in [13] (and further refined in [14]), the precise holographic behavior of the bulk gravity theory is possible because of a UV-IR correspondence between the scale in the boundary theory and the scale in the elementary 
string theory (or M-theory) in the bulk. In the AdS/CFT correspondence, infrared effects in the bulk theory map to ultraviolet effects in the boundary theory [13].

Elementary string theory itself is known to exhibit another UV-IR correspondence, which relates the ultraviolet regime on the string world-sheet to the ultraviolet effects in space-time. One way to see this heuristically is in the logarithmic behavior of the worldsheet propagator, $\langle X(0) X(\sigma)\rangle \sim \log \sigma$. This UV-IR correspondence between the worldsheet of the elementary string and the space-time in which the string propagates should also hold in the Type IIB theory on $\mathrm{AdS}_{5} \times \mathbf{S}^{5}$, at least for large $g_{s} N$. In combination with the UV-IR correspondence of [13], this UV-IR correspondence allows us to derive a direct correspondence of scales between the world-sheet of the string and the space-time of QCD. Thus, we obtain the following UV-UV correspondence:

Ultraviolet effects on the string world-sheet are mapped to ultraviolet effects in QCD.

This behavior is not surprising, and its validity in the hypothetical QCD string theory has indeed been anticipated. Here, however, we have derived this anticipated correspondence of scales in QCD string theory from the microscopic theory, using a very simple chain of arguments based on the AdS/CFT correspondence.

Having argued that the ultraviolet regime on the string world-sheet should map to the ultraviolet effects in QCD, it seems somewhat awkward that the AdS construction leads to a microscopic description of gauge theory in terms of elementary string theory, in which the anticipated UV-UV correspondence is rather obscure. It would seem natural to look for another string theory description, in which the UV-UV correspondence between the string world-sheet and the QCD space-time would be more manifest.

One such theory was proposed a few years ago, in the form of a particular supersymmetrization of the rigid string. This theory was tested in the case of pure-glue QCD in two dimensions, and was found to precisely reproduce the exact results of its large- $N$ expansion previously obtained in [15].

\section{Strings and $\mathrm{QCD}_{2}$}

Two-dimensional pure QCD is an exactly solvable theory [16,17] on any Riemann

surface 2 and for any (compact) gauge group. In the case of $S U(N)$, its large- $N$ expansion was analyzed in [15], and it was shown that the partition function as well as correlation

2 Throughout this paper we will work in Euclidean signature. 
functions of Wilson loops can be expressed as an infinite sum over maps from auxiliary world-sheets to the $1+1$ dimensional space-time. Schematically, the partition function for $\mathrm{QCD}_{2}$ on a surface of genus $G$ can be written in the large- $N$ expansion as [15]

$$
\mathcal{Z}_{G}=\sum_{g} \frac{1}{N^{2 g-2}} \sum_{n, \tilde{n}} e^{-\lambda A(n+\tilde{n}) / 2} \sum_{i=0}^{t}\left(\frac{\lambda A}{2}\right)^{i} \omega_{i, g, G, i, n, \tilde{n}}
$$

Here $\lambda=g_{2}^{2} N$ is the two-dimensional 't Hooft coupling, $A$ is the area of the space-time, and $1 / N$ is the string coupling constant. The string world-sheet covers the space-time $n$ times in the orientation preserving sector, and $\tilde{n}$ times in the orientation reversing sector; $n+\tilde{n}>0$. The upper limit $t$ in the last summation depends on $g, G, n$ and $\tilde{n}$. $\omega$ are numerical coefficients whose precise form can be found in [15].

In [10,12], this exact large- $N$ expansion was found to correspond to the path integral of a new kind of rigid string theory. An alternative approach to this problem was presented in [11]. The results of 10-12 give us the first known example of a complete world-sheet Lagrangian description of QCD string theory.

\subsection{Large- $N$ Expansion in $\mathrm{QCD}_{2}$ as a Rigid String Theory}

In this section we review some of the highlights of the world-sheet representation of $\mathrm{QCD}_{2}$ strings, in the framework of [10,12]. Our discussion will be relatively brief; the reader can find details in [12].

One starts with the crucial observation [10 that in the large- $N$ expansion (3.1), only minimal-area surfaces contribute. The world-sheet theory should therefore be expected to localize to minimal-area maps. Consider maps from a given world-sheet $\Sigma$ with coordinates $\sigma^{a}$ to a fixed space-time manifold with coordinates $x^{\mu}$ and fixed metric $g_{\mu \nu}$ (not necessarily flat). The minimal-area condition

$$
\Delta x^{\mu}=0
$$

where $\Delta$ is the Laplace operator with respect to the induced world-sheet metric $h_{a b}=$ $\partial_{a} x \cdot \partial_{b} x$, is almost empty in two target dimensions, but not quite. Its main purpose is to forbid maps with folds of non-zero length. The only moduli of minimal-area maps are the locations of various singularities of the maps (such as branched points).

There is a well-known technique for constructing path integrals that localize to solutions of a given equation [18]. Following this procedure in the case of the minimal-area 
condition (3.2), one derives a certain supersymmetric world-sheet theory [10,12]. This theory is described by the following world-sheet Lagrangian

$$
\begin{aligned}
\mathcal{L}=\frac{1}{\alpha_{0}} \int_{\Sigma} & d^{2} \sigma \sqrt{h}\left\{\frac{1}{2} \Delta x \cdot \Delta x-\chi \cdot \Delta \psi-R_{\mu \nu \sigma \rho} \chi^{\mu} \psi^{\nu} \chi^{\sigma} \psi^{\rho}\right. \\
& \left.+\left(h^{a b} h^{c d}-h^{a c} h^{b d}-h^{a d} h^{b c}\right) \partial_{a} \chi \cdot \partial_{b} x \partial_{c} \psi \cdot \partial_{d} x+\ldots\right\}
\end{aligned}
$$

where the fermionic fields $\psi^{\mu}$ and $\chi^{\mu}$ are world-sheet scalars, the $\cdot$ denotes the inner product with respect to $g_{\mu \nu}$. We have left out terms of higher order in fields that are not relevant for our discussion here; the full Lagrangian in curved space-time can be found in [12].

In addition to world-sheet diffeomorphism symmetry, the Lagrangian (3.3) is invariant under two mutually anticommuting nilpotent supersymmetries,

$$
\begin{aligned}
{\left[Q, x^{\mu}\right] } & =\psi^{\mu}, & {\left[\bar{Q}, x^{\mu}\right] } & =\chi^{\mu}, \\
\left\{Q, \psi^{\mu}\right\} & =0, & \left\{\bar{Q}, \psi^{\mu}\right\} & =-\Delta x^{\mu}+\ldots, \\
\left\{Q, \chi^{\mu}\right\} & =\Delta x^{\mu}+\ldots, & \left\{\bar{Q}, \chi^{\mu}\right\} & =0 .
\end{aligned}
$$

In the topological interpretation of the theory, $Q$ is of course the BRST charge, while $\bar{Q}$ would be an "accidental" anti-BRST symmetry.

The kinetic term $\sim(\Delta x)^{2}$ in $(3.3)$ is the famous extrinsic curvature term that appears in the rigid string Lagrangian [19] (see also [20 26] for more on the bosonic rigid string). The dimensionless coupling constant $\alpha_{0}$ is usually called the string rigidity. It is rather striking that we have derived, from the sole requirement of localization to minimal-area maps, a certain supersymmetrized version of the rigid string Lagrangian!

The on-shell symmetries (3.4) can easily be promoted into off-shell symmetries of the theory, by reintroducing an auxiliary field $B^{\mu}$, and rewriting the Lagrangian (for simplicity in flat space-time) as

$$
\begin{aligned}
\mathcal{L}=\frac{1}{\alpha_{0}} \int_{\Sigma} & d^{2} \sigma \sqrt{h}\left\{h^{a b} \partial_{a} B \cdot \partial_{b} x+h^{a b} \partial_{a} \psi \cdot \partial_{b} \chi\right. \\
& \left.+\left(h^{a b} h^{c d}-h^{a c} h^{b d}-h^{a d} h^{b c}\right) \partial_{a} \chi \cdot \partial_{b} x \partial_{c} \psi \cdot \partial_{d} x+B^{2} \text { terms }\right\} .
\end{aligned}
$$

The off-shell supersymmetry algebra is identical with (3.4), with the $\Delta x^{\mu}+\ldots$ terms replaced by $B^{\mu}$. Of course, $B^{\mu}$ satisfies $\left[Q, B^{\mu}\right]=\left[\bar{Q}, B^{\mu}\right]=0$.

The ordinary bosonic rigid string Lagrangian also contains a tension term [19]. In our supersymmetric rigid string theory, no tension term is allowed by the off-shell supersymmetry of (3.5). 
Physical states are defined as the cohomology classes of the BRST operator $Q$, with an extra condition of gauge invariance imposed by world-sheet diffeomorphism symmetry. These conditions on physical states leave precisely one bosonic state in each non-trivial winding sector of the closed string, in accord with the known spectrum of states in $\mathrm{QCD}_{2}$ string theory.

Almost by construction (or more precisely, by world-sheet supersymmetry (3.4)), the path integral localizes to the moduli space of minimal-area maps. One can calculate the path integral in the semi-classical, $\alpha_{0} \rightarrow 0$ limit of the world-sheet theory. The partition function will not depend on $\alpha_{0}$. It is not difficult to see that the path integral in fact calculates the Euler number of the moduli spaces of minimal-area maps [10,12]. Indeed, recall that the zero modes of $\psi^{\mu}$ behave as one-forms on the moduli spaces. Upon integration over the zero modes of $\chi^{\mu}$ and $\psi^{\mu}$, the curvature-dependent term in (3.3) will give the Euler number density on the moduli space.

It was conjectured in [10] that the coefficients in the large- $N$ expansion (3.1) at $\lambda A=0$ are precisely the Euler numbers of the moduli spaces of minimal-area maps, and that the path integral in the topological rigid string theory reproduces the exact results of the large- $N$ expansion of $\mathrm{QCD}_{2}$. The Euler numbers of the relevant moduli spaces were first obtained in [11], and the conjecture was later proven in [12].

The case of non-zero $\lambda A$ is a little more involved, but it can also be analyzed in the framework of topological string theory. The structure of the large- $N$ expansion (3.1) indicates that the corresponding string theory is still localized to minimal-area maps, and suggests that the polynomial area dependence arises from measuring the volume of various components of the moduli space. This can be represented in the topological rigid string as follows. Due to localization to moduli spaces, one can calculate correlation functions of observables that correspond to universal cohomology classes on the moduli spaces; a particular linear combination of these universal observables then corresponds to the tension term. The exponential dependence on $A$ in (3.1) is reproduced from the evaluation of $\int_{\Sigma} d^{2} \sigma \sqrt{h}$ on the minimal-area map. For details on the perturbative treatment of the $\lambda A \neq 0$ case in the topological rigid string theory, the reader is again referred to [12]. We will have more to say about the area dependence in $\mathrm{QCD}_{2}$ string theory in Section 4.5.

\subsection{AdS Construction for $\mathrm{QCD}_{2}$}

Using the AdS/CFT correspondence, and following the construction of $\mathrm{QCD}_{4}$ in [4], we could in principle construct $\mathrm{QCD}_{2}$ in several ways. One option is to start with $N$ 
M2-branes at finite temperature, i.e., with the superconformal theory in three dimensions at the strongly-coupled fixed point with $S O(8)$ R-symmetry, compactified on an $\mathbf{S}^{1}$ with supersymmetry-breaking periodicity conditions. According to the AdS/CFT correspondence, this theory would correspond to the Schwarzschild black hole in the $\mathrm{AdS}_{4}$ space-time (times the internal $\mathbf{S}^{7}$ ).

Here we will start instead with $\mathcal{N}=4$ super Yang-Mills in four dimensions, and compactify this theory, first to three dimensions on $\mathbf{S}^{1}$ with radius $R_{1}$ and supersymmetrypreserving conditions, and subsequently to two dimensions on $\mathbf{S}^{1}$ with radius $R_{2}$ and supersymmetry-breaking conditions. The first step leads to dimensionally reduced YangMills theory with sixteen supercharges, at distances much larger than $R_{1}$. Keeping $R_{2} \gg$ $R_{1}$, and looking at the system at distances much larger than $R_{2}$ in the remaining two noncompact dimensions, the second step should give masses of order $1 / R_{2}$ to the fermions, and of order $g_{4}^{2} N / R_{2}$ to the scalars.

Since we would like to compare the theory to $\mathrm{QCD}_{2}$ on a two-torus of finite area $A$, we will further compactify the two remaining dimensions on a two-torus with both radii of order $L=\sqrt{A}$ (say on a rectangular torus with both radii equal to $L$ ). The radius $L$ of the two-torus sets the scale in $\mathrm{QCD}_{2}$.

In order to isolate $\mathrm{QCD}_{2}$ we want to keep $L$ fixed while sending $R_{1} \ll R_{2} \rightarrow 0$. The $\mathrm{QCD}_{2}$ coupling $g_{2}$ is related to the four-dimensional super Yang-Mills coupling via $\left(2 \pi^{2}\right) g_{2}^{2}=g_{4}^{2} / R_{1} R_{2}$. The two-dimensional 't Hooft coupling $\lambda=g_{2}^{2} N$ is then given in terms of the four-dimensional quantities by

$$
\lambda=\frac{g_{4}^{2} N}{(2 \pi)^{2} R_{1} R_{2}} .
$$

We are interested in the physics at scales of order $L$, in the asymptotic large- $N$ expansion, and at zero QCD string tension $\lambda$. Thus, we want to take the limit where $L$ is fixed, $R_{1}, R_{2} \rightarrow 0$, and $N \gg 1$ but finite.

In order to make contact with the supersymmetric rigid string description of $\mathrm{QCD}_{2}$, we are interested in the limit of $\lambda \rightarrow 0$, in which as we have seen in the previous section the string path integral representation is most natural. $\mathrm{QCD}_{2}$ on a torus is not completely trivial even in the tensionless limit $\lambda A=0$, as one can study correlation functions of Wilson lines. However, it is still convenient to keep $\lambda A$ small but non-zero, as a regulator in the sum over world-sheets of various winding numbers $n, \tilde{n}$ in (3.1).

Thus, we need $\lambda$ to be small in units set by $L$, which means that $g_{4}$ will have to scale with $R_{1}$ and $R_{2}$ as $g_{4}^{2} \sim R_{1} R_{2}$. In order to keep $\lambda$ small in the infinite $N$ limit, $g_{4}$ will 
have to scale with $N$ as well: $g_{4}^{2} \sim R_{1} R_{2} / N$. The AdS/CFT relation identifies $(2 \pi)^{-1} g_{4}^{2}$ with the Type IIB string coupling constant $g_{s}$, and we see that in the limit of our interest the Type IIB string theory is indeed very weakly coupled.

As $R_{1}$ and $R_{2}$ go to zero at fixed small $\lambda$, the masses of fermions and scalars go to inifinity, and the states should indeed decouple. The effective bare coupling at the compactification radius is $g_{2}^{2} N(2 \pi)^{2} R_{1} R_{2}=g_{4}^{2} N$, which is also small in our limit.

The five-dimensional metric of the corresponding Type IIB string theory is

$$
d s^{2}=\left(\frac{\rho^{2}}{b^{2}}-\frac{b^{2}}{\rho^{2}}\right) d \tau^{2}+\frac{d \rho^{2}}{\left(\frac{\rho^{2}}{b^{2}}-\frac{b^{2}}{\rho^{2}}\right)}+\rho^{2} \sum_{i=1}^{3} d x_{i}^{2}
$$

The period of $\tau$ is $\beta_{1}=\pi b, b$ is related to the Type IIB string coupling $g_{s}$, the string scale $\ell_{s}$ and the RR flux $N$ by $2 b=\ell_{s}\left(4 \pi g_{s} N\right)^{1 / 4}$, and we have temporarily suppressed the $\mathbf{S}^{5}$ part of the full Type IIB string theory.

It will be convenient to rescale $\tau$ so that the rescaled coordinate $\theta=\ell_{s}^{-1}\left(4 \pi g_{s} N\right)^{-1 / 4} \tau$ has period $2 \pi$. We also introduce a rescaled coordinate $r=2 \rho / b$, and the metric becomes

$$
d s^{2} / \ell_{s}^{2}=\left(4 \pi g_{s} N\right)^{1 / 2}\left(r^{2}-\frac{1}{r^{2}}\right) d \theta^{2}+\left(4 \pi g_{s} N\right)^{1 / 2} \frac{d r^{2}}{\left(r^{2}-\frac{1}{r^{2}}\right)}+\left(4 \pi g_{s} N\right)^{1 / 2} r^{2} \sum_{i=1}^{3} d x_{i}^{2}
$$

So far we have compactified only one dimension in the boundary field theory, on a circle with supersymmetry-breaking boundary conditions. We also want to compactify the remaining Killing dimensions $x_{i}$ on a three-torus, with one dimension (say $x_{3} \equiv y$ ) smaller than $\tau$, and the two remaining $x_{1,2}$ much larger than $\tau$. The radius of $\theta$ at infinity can of course be arbitrarily changed by rescaling $r$ and defining the boundary metric as $r^{-2} d s^{2}$ in the $r \rightarrow 0$ limit.

We obtain the correct compactification by requiring that the radius of $y$ at infinity be $R_{2} / R_{1}$ times the radius of $\theta$ at infinity (and similarly for $x_{i}$ ). It will again be convenient to rescale $y$ and $x_{i}$ such that the rescaled variables $Y$ and $X_{i}$ have period $2 \pi$. In terms of these variables, the fully compactified metric is now

$$
\begin{aligned}
d s^{2} / \ell_{s}^{2}=\left(4 \pi g_{s} N\right)^{1 / 2} & \left(r^{2}-\frac{1}{r^{2}}\right) d \theta^{2}+\left(4 \pi g_{s} N\right)^{1 / 2} \frac{d r^{2}}{\left(r^{2}-\frac{1}{r^{2}}\right)} \\
& +\left(4 \pi g_{s} N\right)^{1 / 2} r^{2}\left(\left(\frac{R_{2}}{R_{1}}\right)^{2} d Y^{2}+\left(\frac{L}{R_{1}}\right)^{2} \sum_{i=1}^{2} d X_{i}^{2}\right)
\end{aligned}
$$


Now we are ready to study this metric in the limit that should isolate only the $Q C D_{2}$ degrees of freedom. We have argued that in this limit, as $R_{1}$ and $R_{2}$ go to zero, the Type IIB string coupling is given by

$$
g_{s}=2 \pi \frac{\lambda R_{1} R_{2}}{N} .
$$

Using this formula, one can see that all dependence on $N$ disappears from the metric, which now becomes

$$
\begin{aligned}
\frac{d s^{2}}{2(2 \pi)^{2} \ell_{s}^{2}}=\lambda^{1 / 2}\left(R_{1} R_{2}\right)^{1 / 2}\left(r^{2}-\frac{1}{r^{2}}\right) & d \theta^{2}+\lambda^{1 / 2}\left(R_{1} R_{2}\right)^{1 / 2} \frac{d r^{2}}{\left(r^{2}-\frac{1}{r^{2}}\right)} \\
& +\lambda^{1 / 2} r^{2}\left(\frac{R_{2}^{5 / 2}}{R_{1}^{3 / 2}} d Y^{2}+\frac{L^{2} R_{2}^{1 / 2}}{R_{1}^{3 / 2}} \sum_{i=1}^{2} d X_{i}^{2}\right) .
\end{aligned}
$$

In this picture, $N$ appears only in the expression for the string coupling (3.10), leading to the $g_{s} \sim 1 / N$ relation anticipated in QCD string theory.

We will take the "continuum $\mathrm{QCD}_{2}$ " limit by sending $R_{2} \equiv R$ to zero while keeping $R_{1} / R_{2} \ll 1$ fixed. In this limit, the metric scales with $R \rightarrow 0$ as follows,

$$
\frac{d s^{2}}{2(2 \pi)^{2} \ell_{s}^{2}}=\lambda^{1 / 2} R\left(r^{2}-\frac{1}{r^{2}}\right) d \theta^{2}+\lambda^{1 / 2} R \frac{d r^{2}}{\left(r^{2}-\frac{1}{r^{2}}\right)}+\lambda^{1 / 2} R r^{2} d Y^{2}+\lambda^{1 / 2} \frac{L^{2}}{R} r^{2} \sum_{i=1}^{2} d X_{i}^{2} .
$$

As $R$ goes to zero, only the two dimensions corresponding to the space-time of $\mathrm{QCD}_{2}$ are large. All the remaining dimensions (including the $\mathbf{S}^{5}$ that is implicit in our formulas) are suppressed by a positive power of $R$. Of course, we still have $1 \leq r \leq \infty$.

This little construction of $\mathrm{QCD}_{2}$ from the AdS/CFT correspondence illustrates one of the main points that we want to make: decoupled QCD in the continuum limit corresponds to a rather singular limit on the AdS side of the AdS/CFT correspondence, a limit in which supergravity is manifestly invalid. This conclusion is fairly independent of the space-time dimension, and applies equally well to the construction of continuum $\mathrm{QCD}_{4}$ from the six-dimensional $(0,2)$ theory compactified on a two-torus [4], even though our reason for sending $\lambda \rightarrow 0$ in that case is different [7].

As we have argued in the Introduction, continuum QCD might be described by elementary string theory only in this singular limit, which is manifestly hard to understand. We may therefore want to describe it directly by another string theory that is more suitable for the description of the surviving degrees of freedom in that singular limit of Type IIB string theory. 


\subsection{Comparison}

In the case of $\mathrm{QCD}_{2}$, we already have an answer for the world-sheet theory of QCD strings, in the form of the supersymmetric rigid string reviewed in Section 3.1. In conjunction with the AdS construction of $\mathrm{QCD}_{2}$ presented in Section 3.2, this leads to the following conjecture:

The two-dimensional supersymmetric rigid string theory of [10,19] is dual to the elementary Type IIB string theory compactified on the black-hole geometry asymptotic to $\mathrm{AdS}_{5} \times \mathbf{S}^{5}$ in the singular limit described in Section 3.2.

In the sense of this conjecture, the supersymmetric rigid string theory of $\mathrm{QCD}_{2}$ represents a "boundary condition" on any candidate QCD string theory that we may want to derive from Type II string theory on the singular backgrounds that correspond to the continuum limit of QCD in higher dimensions.

The supersymmetric rigid string theory belongs to the category of string theories whose kinetic term is given by the extrinsic curvature term $\left(\Delta x^{\mu}\right)^{2}$. Such theories exhibit a UV-UV correspondence between the world-sheet of the string and the space-time in which the string propagates. One can see this fact simply from the structure of the Lagrangian for $x^{\mu}$ at small $\alpha_{0}$. Due to the higer-order form of the kinetic term, $x^{\mu}$ has the canonical dimension of length on the world-sheet. This should again be contrasted with elementary string theory, which exhibits the UV-IR correspondence.

The supersymmetric rigid string was constructed from the requirement of localization to moduli spaces of minimal-area surfaces. This localization has an intriguing analogy in the AdS construction of $\mathrm{QCD}_{2}$. In the AdS construction, the elementary string tension is related to the effective string tension, as seen in the Wilson loop average, by a factor of $b^{2}$ [27,7]. As we send $b$ to zero and keep the effective string tension (small but) finite in the units set by $L$, the elementary string tension goes to infinity. Thus, the elementary string is classical on the world-sheet, and its world-sheet path integral should be dominated by its classical solutions.

In the path integral of the supersymmetric rigid string, one of the main subtleties comes from the fact that any minimal-area map between two-dimensional surfaces in a generic homotopy class exhibits singularities where the induced metric is degenerate. Due to this singular nature of generic minimal-area maps, a more precise definition of the class

of surfaces that contribute to the path integral is needed. The correct choice is the class of so-called stratified surfaces [12]. A related phenomenon has been observed in [7], where the 
calculation of Wilson lines in the supergravity regime leads to collapsed minimal surfaces with two disk components connected by an infinitesimally thin tube, which is - at scales large compared to the string scale - a typical stratified surface. Using the conjectured relation between the Type IIB string theory and the supersymmetric rigid string, the full Type IIB string theory on the background slightly away from its singular limit should provide a covariant regularization of this problem of singular world-sheets in the path integral of the supersymmetric rigid string.

It would be extremely interesting to see whether the supersymmetric rigid string theory can be derived from the Type IIB string theory sigma model in the corresponding singular limit, once a precise formulation of the latter becomes available at least in the limit of large 't Hooft coupling.

\section{Rigid String Theory with Parisi-Sourlas Supersymmetry}

Given that the supersymmetric rigid string approach has been successful in describing $\mathrm{QCD}_{2}$ strings, one is naturally interested in possible generalizations of the theory to dimensions greater than two. The purpose of this Section is to show that at least one natural extension of the supersymmetric rigid string theory does indeed exist. This rigid string theory is characterized by local Parisi-Sourlas supersymmetry on the world-sheet. We will see that in dimensions greater than two, this theory exhibits very interesting dynamics, involving asymptotic freedom and dimensional transmutation. Consequently, the theory is effectively topological only in two space-time dimensions, where it reproduces the $\mathrm{QCD}_{2}$ string theory reviewed in Section 3.1.

Some ideas presented in this Section have also been advocated independently by Polyakov ([6,28] and unpublished).

\subsection{A Superspace Formulation}

Our starting point will be the supersymmetric rigid string Lagrangian (3.5), in flat space-time $\mathbf{R}^{D}$.

The rigid string Lagrangian (3.5) can be succinctly rewritten in a superspace form if we assemble all the fields in the Lagrangian into $D$ superfields,

$$
X^{\mu}\left(\sigma^{a}, \theta, \bar{\theta}\right)=x^{\mu}+\theta \psi^{\mu}+\bar{\theta} \chi^{\mu}+\theta \bar{\theta} B^{\mu}
$$


Here $\theta, \bar{\theta}$ (which we will sometimes collectively denote by $\theta^{\alpha}$ ) are two fermionic coordinates that transform as scalars with respect to ordinary world-sheet diffeomorphisms. 3

In this notation, we can write the Lagrangian of the topological rigid string as a sum of the following two terms, $\mathcal{L}_{1}$ and $\mathcal{L}_{2}$. The first term, $\mathcal{L}_{1}$, looks like the ordinary bosonic Nambu-Goto Lagrangian, with the target coordinates replaced by the superfields $X^{\mu}$, and integrated over the whole super world-sheet:

$$
\mathcal{L}_{1}=\frac{1}{\alpha_{0}} \int_{\Sigma} d^{2} \sigma d^{2} \theta \sqrt{\operatorname{det} H_{a b}}
$$

Here $d^{2} \theta \equiv d \bar{\theta} d \theta$, and $H_{a b} \equiv H_{a b}\left(\sigma^{a}, \theta^{\alpha}\right)$ is the induced metric on the world-sheet:

$$
H^{a b} \equiv \partial_{a} X^{\mu} g_{\mu \nu} \partial_{b} X^{\nu} \equiv \partial_{a} X \cdot \partial_{b} X
$$

This Lagrangian is invariant under a supersymmetric extension of the world-sheet diffeomorphism group. It is not, however, invariant under all superdiffeomorphisms of the super world-sheet. The group of symmetries of the Lagrangian consists of arbitrary reparametrizations of $\sigma^{a}$,

$$
\tilde{\sigma}^{a}=\tilde{\sigma}^{a}\left(\sigma^{b}, \theta, \bar{\theta}\right), \quad \tilde{\theta}^{\alpha}=\theta^{\alpha},
$$

and all ( $\sigma^{a}$-independent) diffeomorphisms of $\theta^{\alpha}$,

$$
\tilde{\theta}^{\alpha}=\tilde{\theta}^{\alpha}\left(\theta^{\beta}\right)
$$

Hence, although the new bosonic coordinates $\tilde{\sigma}^{a}$ can be arbitrary regular functions of both $\sigma^{a}$ and $\theta^{\alpha}$, the new fermionic coordinates $\tilde{\theta}^{\alpha}$ are $\sigma^{a}$-independent functions of $\theta^{\beta}$ only. This "semirigid supersymmetry" [12] arises quite naturally in topological field theories with diffeomorphism invariance, as it is the smallest group that combines global BRST symmetry with diffeomorphisms.

So far we have found the superspace expression for only a part of the Lagrangian of the topological rigid string. The requirement of invariance under semirigid world-sheet supersymmetry gives us one more building block for the construction of Lagrangians. It is easy to see that

$$
S \equiv \partial_{\theta} X \cdot \partial_{\bar{\theta}} X-\partial_{\theta} X \cdot \partial_{a} X H^{a b} \partial_{b} X \cdot \partial_{\bar{\theta}} X
$$

3 i.e., they form a trivial two-dimensional bundle over $\Sigma$, with odd fiber. Since this trivial bundle is unique, we will not distinguish in our notation between $\Sigma$ and its super-extension. 
is invariant under the semirigid supersymmetry (4.4), (4.5). Probably the easiest way to see this invariance is to introduce the induced supermetric on the super world-sheet (here we introduce the superspace index on the world-sheet, $A \equiv(a, \alpha)$, with $a=1,2, \alpha=1,2)$ :

$$
H_{A B}=(-1)^{B} \partial_{A} X \cdot \partial_{B} X,
$$

and notice that $S$ is the inverse of the $\theta \bar{\theta}$ component of $H^{A B}$, where $H^{A B}$ is the inverse of the induced supermetric,

$$
S=\left(H^{\theta \bar{\theta}}\right)^{-1} .
$$

Invoking the transformation properties of the supermetric, $S$ is invariant under semirigid superdiffeomorphisms. We can use this invariant to write

$$
\mathcal{L}_{2}=\frac{1}{\alpha_{0}} \int_{\Sigma} d^{2} \sigma d^{2} \theta \sqrt{\operatorname{det} H_{a b}} S .
$$

While the $\theta \bar{\theta}$ component of $S$ produces the $B^{2}$ term of the topological rigid string Lagrangian (3.5).

Thus, we have succeeded in rewriting the theory of Section 3.1 as a rigid string theory with semirigid supersymmetry. The semirigid supersymmetry algebra contains two scalar mutually anticommuting supercharges $Q$ and $\bar{Q}$ of (3.4) which act on the superfields (4.1) by

$$
Q=\frac{\partial}{\partial \theta}, \quad \bar{Q}=\frac{\partial}{\partial \bar{\theta}} .
$$

This type of supersymmetry, known in the literature as the Parisi-Sourlas supersymmetry [29-31], is a very universal symmetry of many physical systems. It has a remarkably broad range of applications, in the physics of polymers, quenched disorder, systems in random external fields, spin glasses, and stochastic quantization, to name just a few. (See e.g. 29-39] for some references on this subject.) In particular, the way in which our complicated higher-order rigid string Lagrangian (3.3) can be rewritten in the Parisi-Sourlas superspace in terms of a supersymmetrized Nambu-Goto Lagrangian (4.3) (and (4.9)) is also very reminiscent of similar constructions in theories with Parisi-Sourlas supersymmetry. In the case of our rigid string, Parisi-Sourlas supersymmetry has been combined with diffeomorphism invariance, into the semirigid supersymmetry of the theory.

Having recognized that our theory is in fact a string theory with world-sheet ParisiSourlas supersymmetry, we now want to write down the most general world-sheet Lagrangian that respects the symmetry (together with world-sheet diffeomorphisms and space-time Poincaré invariance) and contains only marginal or relevant terms. 
The structure of the Lagrangian (4.2) near the gaussian fixed point at $\alpha_{0}=0$ determines canonical world-sheet dimensions as follows. $d \sigma^{a}$ has the dimension of world-sheet length, as does $\theta^{\alpha}$. Since $d \theta \sim \partial \theta$, the super-volume element $d^{2} \sigma d^{2} \theta$ is dimensionless. All superfields $X^{\mu}$ have the dimension of world-sheet length, and $\alpha_{0}$ is indeed dimensionless.

With this counting, $S$ of (4.6) is of canonical dimension zero, and the general theory is described by the following Lagrangian:

$$
\mathcal{L}=\int_{\Sigma} d^{2} \sigma d^{2} \theta \sqrt{\operatorname{det} H_{a b}}\left(\frac{1}{\alpha_{0}}+\sum g_{i 0} S^{i}\right)
$$

where the sum goes over all positive integer powers of $S$, and $g_{i 0}$ are independent coupling constants. There are no relevant terms that would respect the symmetries.

At first glance, it might seem that in (4.11) we have a theory with an infinite number of canonically marginal coupling constants $g_{i 0}$; however, the number of independent ones is finite since $S^{n}$ vanishes for $n$ high enough. The analysis of this Lagrangian at the quantum level and the identification of fixed points in the space of all independent couplings represents a very interesting but rather involved problem, which will not be addressed here in full generality. Instead, we will limit the number of independent couplings in (4.11) by imposing further symmetry on the theory.

Note that in the component form of the general Lagrangian (4.11) with arbitrary choice of the bare couplings, $B^{\mu}$ always comes out as a Gaussian auxiliary field, and can be integrated out exactly. Indeed, neither higher powers of $B^{\mu}$ nor its world-sheet derivatives can emerge in the Lagrangian, since only the first derivatives of $X^{\mu}$ are allowed by dimension counting; in other words, the higher powers of $B^{\mu}$ as well as its derivatives are always irrelevant.

\subsection{Theory with Local Parisi-Sourlas Supersymmetry}

So far we have discussed a theory whose symmetry is given by the semi-rigid ParisiSourlas supersymmetry (4.4), (4.5). In this Section we go one step further, and enhance the gauge symmetries so that they will permit only one marginal term (and no relevant terms) in the world-sheet Lagrangian. Upon partial gauge fixing, this theory with enlarged supersymmetry will belong to the class of theories (4.11) discussed in Section 4.1.

We extend the supersymmetries (4.4), (4.5) to all super-diffeomorphisms of the super world-sheet $\left(\sigma^{a}, \theta^{\alpha}\right)$. In order to keep in contact with the general class of theories (4.11), we will use the following trick. First, extend the space-time to a supermanifold by introducing 
two fermionic dimensions, with coordinates $\vartheta^{m}, m=1,2$ (we will also use the simpler notation $\vartheta^{1} \equiv \vartheta$ and $\vartheta^{2} \equiv \bar{\vartheta}$ ). The space-time manifold is now parametrized by

$$
y^{M} \equiv\left(x^{\mu}, \vartheta^{m}\right)
$$

The flat space-time metric $G_{\mu \nu}=\delta_{\mu \nu}$ is extended to a super-metric $G_{M N}=\left(\delta_{\mu \nu}, \varepsilon_{m n}\right)$. The embedding of the world-sheet to the space-time is described by world-sheet superfields, which we will denote by capital letters:

$$
Y^{M} \equiv\left(X^{\mu}, \Theta^{m}\right)
$$

The component expansion of $X^{\mu}$ is that of (4.1).

We require that the theory be gauge invariant under all world-sheet superdiffeomorphisms, and globally invariant under the space-time super-Poincaré symmetry $\operatorname{IOSp}(D \mid 2)$. The simplest Lagrangian with these symmetries is

$$
\mathcal{L}=\frac{1}{\alpha_{0}} \int_{\Sigma} d^{2} \sigma d^{2} \theta \sqrt{\operatorname{sdet} H_{A B}}
$$

where $A, B$ goes through all four indices of the supersymmetric world-sheet, and sdet $H_{A B}$ is the superdeterminant of the induced supermetric

$$
H_{A B} \equiv(-1)^{B(B+N)} \partial_{A} Y^{M} G_{M N} \partial_{B} Y^{N} .
$$

No relevant terms and no other marginal terms are allowed by local world-sheet supersymmetry and space-time super-Poincaré invariance.

This theory indeed fits into the general class (4.11), once we partially fix the gauge so that the remaining gauge symmetry is the semirigid supersymmetry of (4.4), 4.5). To get a theory invariant under semirigid diffeomorphisms, we can fix the fermionic part of the gauge symmetry by imposing a Monge gauge in the fermionic dimensions:

$$
\Theta=\theta, \quad \bar{\Theta}=\bar{\theta} .
$$

This gauge fixing reduces the superdeterminant of the induced supermetric to

$$
\operatorname{sdet} H_{A B}=\operatorname{det} H_{a b}\left(\frac{1}{1-S}\right)^{2},
$$

and the Lagrangian (4.15) becomes a particular example of a theory from the general class (4.11), with all coupling constants $g_{i 0}$ fixed uniquely in terms of one coupling, $\alpha_{0}$. 
It is interesting to notice that in the theory with fully local Parisi-Sourlas supersymmetry, the free-field fixed point at small $\alpha_{0}$ does not fix the canonical dimension of the superfields $X^{\mu}, \Theta^{m}$. The full Lagrangian (4.14) before gauge fixing is invariant under the space-time scale transformations $Y^{M} \rightarrow \lambda Y^{M}$ that act trivially on the world-sheet. This additional symmetry is not respected by the gauge fixing, which implicitly assumes $[\Theta]=[\theta]=-1$. We could keep track of this symmetry during the gauge fixing by introducing a dimensionful parameter $\gamma$, and rewriting the Monge gauge as $\Theta=\gamma \theta, \bar{\Theta}=\gamma \bar{\theta}$. This parameter $\gamma$ has the dimension of space-time length and world-sheet mass.

Another interesting fact about the fermionic Monge gauge is that it identifies translations along the fermionic dimensions on the world-sheet with supertranslations in the extended space-time. In the topological interpretation of the world-sheet Lagrangian, world-sheet supertranslations correspond to the topological BRST (and anti-BRST) symmetry (3.4).

\subsection{Asymptotic Freedom}

The symmetries of our theory permit only one marginal coupling, the string rigitity $\alpha_{0}$. In this section we will consider the weak-coupling expansion in $\alpha_{0}$, and calculate the one-loop beta function. We will closely follow the spirit of [40] and in particular [41], where a detailed analysis of the bosonic rigid string path integral has been performed. Some additional information on the bosonic case can also be found in [42, 43, 22,

It will be convenient to work in the off-shell, superfield formalism developed in Section 4.1. Split the fields into a reference background plus fluctuations, $Y^{M}=$ $\bar{Y}^{M}+\sqrt{\alpha_{0}} \delta Y^{M}$. We first keep $\bar{Y}^{M}$ arbitrary, but eventually we will evaluate the oneloop path integral for a conveniently chosen $\bar{Y}^{M}$.

Choose normal gauge with respect to the reference surface $\bar{Y}^{M}$,

$$
\partial_{A} \bar{Y} \cdot \delta Y=0
$$

The first contribution to the effective action will come from the determinant of the operator in the quadratic expansion of the Lagrangian in $\delta Y^{M}$ 's,

$$
\frac{1}{\alpha_{0}} \int_{\Sigma} d^{2} \sigma d^{2} \theta \sqrt{\operatorname{sdet} H_{A B}(\bar{Y})}+\int_{\Sigma} d^{2} \sigma d^{2} \theta \delta Y^{M} \mathcal{O}_{M N} \delta Y^{N}
$$

Here $\mathcal{O}$ is the second-order operator density on the world-sheet, essentially the superLaplace operator on the normal bundle in the reference background $\bar{Y}^{M}$. 
There are several other contributions to the effective action, and we have to investigate whether they can contribute to the one-loop beta function.

First, the Faddeev-Popov determinant corresponding to our choice of gauge is not trivial [41]. Still, this determinant is independent of $\alpha_{0}$, and to first order in $\alpha_{0}$, we can simply evaluate it in the reference background $\bar{Y}^{M}$. In this background, the determinant is given by the superdeterminant of the world-sheet supermetric,

$$
\Delta_{\mathrm{FP}} \sim\left(\operatorname{sdet} H_{A B}(\bar{Y})\right)^{-1} .
$$

Since the result is ultralocal in $\left(\sigma^{a}, \theta^{\alpha}\right)$, it will not contribute to the renormalization of $\alpha_{0}$. All fluctuations will be of higher order in $\alpha_{0}$, and thus the FP determinant will not contribute to the one-loop beta function.

The last contribution to the effective action comes from the careful analysis of the measure. In general, the measure is non-linear, and $\delta Y^{M}$-dependent. One can replace the full non-linear measure $[d \delta Y]$ by a flat measure $[d \delta Y]_{0}$, defined with respect to some flat reference metric on the world-sheet. This changes the density of degrees of freedom, which has to be compensated by a renormalization factor in the measure,

$$
[d \delta Y] e^{-\int \mathcal{L}}=[d \delta Y]_{0} e^{-\int \mathcal{L}-\int \delta \mathcal{L}} .
$$

Being a renormalization factor, this Liouville term $\delta \mathcal{L}$ depends on the parameters of $\mathcal{L}$.

For the purpose of our gaussian integration, however, we do not need to know the exact form of the Liouville term. In particular, we do not need the measure, just the metric on the space of fields. The metric given by the full non-linear measure $[d \delta Y]$ coincides with the metric defined with respect to the fixed reference background $\bar{Y}^{M}$. Thus, we do not have to worry about the Liouville term in our one-loop calculation of the beta function.

The terms in the effective action that contribute to the renormalization of $\alpha_{0}$ are thus

$$
\mathcal{L}_{\text {eff }}=\frac{1}{\alpha_{0}} \int_{\Sigma} d^{2} \sigma d^{2} \theta \sqrt{\operatorname{sdet} H_{A B}(\bar{Y})}+\frac{1}{2} \operatorname{str} \bar{H} \ln \mathcal{O},
$$

where $\operatorname{str} \bar{H}$ is the supertrace with respect to the background supermetric, $\operatorname{str} \bar{H} \ldots=$ $\int_{\Sigma} d^{2} \sigma d^{2} \theta \sqrt{\operatorname{sdet} H_{A B}(\bar{Y})} \ldots$

Having identified all possible contributions to the one-loop beta function of $\alpha_{0}$, we identify the renormalized coupling $\alpha$ as the inverse of the coefficient in front of $\int_{\Sigma} d^{2} \sigma d^{2} \theta \sqrt{\operatorname{sdet} H_{A B}(\bar{Y})}$ in the effective action. As we have argued, the $\operatorname{str} \bar{H} \ln \mathcal{O}$ term is the only term that contributes to the renormalization of $\alpha_{0}$. 
So far our argument has been independent of the particular choice of $\bar{Y}^{M}$. Now we will calculate the effective coupling by evaluating the contributions in a convenient background. Notice that the area term $\int_{\Sigma} d^{2} \sigma d^{2} \theta \sqrt{\operatorname{sdet} H_{A B}(\bar{Y})}$ has already been factorized from our definition of the renormalized coupling $\alpha$. This allows us to conveniently calculate $\alpha$ by choosing for $\bar{Y}^{M}$ the flat super world-sheet, thus obtaining

$$
\frac{1}{\alpha(\Lambda)}=\frac{1}{\alpha\left(\Lambda_{0}\right)}+\frac{D-2}{2} \int_{\Lambda_{0}}^{\Lambda} \frac{d^{2} k d^{2} \kappa}{(2 \pi)^{2}} \ln \left(k^{2}-2 \kappa \bar{\kappa}\right),
$$

where $k_{a}, \kappa_{\alpha}$ are the Fourier super-momenta on the flat super world-sheet. uate the momentum integral using the superfield expansion of the logarithm,

$$
\int_{\Lambda_{0}}^{\Lambda} \frac{d^{2} k d^{2} \kappa}{(2 \pi)^{2}} \ln \left(k^{2}-2 \kappa \bar{\kappa}\right)=\int_{\Lambda_{0}}^{\Lambda} \frac{d^{2} k d^{2} \kappa}{(2 \pi)^{2}}\left(\ln k^{2}-2 \frac{\kappa \bar{\kappa}}{k^{2}}\right)=-\frac{1}{\pi} \ln \left(\Lambda / \Lambda_{0}\right) .
$$

Notice that our superspace calculation is a calculation with a supersymmetric cutoff. In the integration over the shell in the momentum space, the momentum modes of the four components of the superfield $Y^{M}$ are all cut off at the same lower and upper scales $\Lambda_{0}$ and $\Lambda$.

To first order in $\alpha_{0}$, (4.23) and (4.24) gives the effective coupling $\alpha(p)$

$$
\alpha(p)=\frac{\alpha_{0}}{1-\frac{D-2}{4 \pi} \alpha_{0} \ln \left(\frac{\Lambda^{2}}{p^{2}}\right)} .
$$

Thus, we have demonstrated to order $\alpha_{0}$ that the theory is asymptotically free in dimensions $D>2$, and $D=2$ is the critical dimension.

\subsection{Quantum Theory at Large D}

We have seen that in dimensions greater than two, the theory is asymptotically free. Non-perturbative effects in $\alpha_{0}$ will be very important in the long-distance dynamics of the theory. To shed some light on the non-perturbative behavior of the model, we will analyze the theory in the mean field approximation at large number of space-time dimensions. Large- $D$ behavior of the bosonic rigid string was studied in [22, 43].

The Lagrangian that will be convenient to use in the $1 / D$ expansion is

$$
\begin{aligned}
\mathcal{L}= & \frac{1}{\alpha_{0}} \int_{\Sigma} d^{2} \sigma d^{2} \theta \sqrt{\operatorname{sdet} H_{A B}} \\
& \quad-\frac{i}{\alpha_{0}} \int_{\Sigma} d^{2} \sigma d^{2} \theta \lambda^{A B}\left\{H_{A B}-(-1)^{B(B+N)} \partial_{A} Y^{M} G_{M N} \partial_{B} Y^{N}\right\} .
\end{aligned}
$$

4 For the super Fourier transform on Parisi-Sourlas superspace, see e.g. 33]. 
Now we treat $H_{A B}$ as an independent supermetric on the world-sheet, and $\lambda^{A B}$ is the Lagrange multiplier that identifies $H_{A B}$ classically with the induced supermetric.

The effective action of the model in the mean field approximation at large $D$ can be calculated as follows. We again split $Y^{M}$ into the classical background part and a quantum part,

$$
Y^{M}=Y_{0}^{M}+\sqrt{\alpha_{0}} Y_{q u}^{M}
$$

The Lagrangian is quadratic in $Y^{M}$ and we can integrate over $Y_{q u}^{M}$ exactly. This gives

$$
\begin{aligned}
\mathcal{L}_{\text {eff }}=\frac{1}{\alpha_{0}} & \int_{\Sigma} d^{2} \sigma d^{2} \theta \sqrt{\operatorname{det} H_{A B}} \\
& -\frac{i}{\alpha_{0}} \int_{\Sigma} d^{2} \sigma d^{2} \theta \lambda^{A B}\left\{H_{A B}-(-1)^{B(B+N)} \partial_{A} Y_{0}^{M} G_{M N} \partial_{B} Y_{0}^{N}\right\} \\
& +\frac{1}{2} \operatorname{str}_{H} \ln \left(\frac{i}{\sqrt{\operatorname{sdet} H_{A B}}} \partial_{A} \lambda^{B A} \partial_{B}\right) .
\end{aligned}
$$

We still have $D$ background fields $Y_{0}^{M}$ explicitly in the Lagrangian, but since they only enter through their induced metric $H_{A B}\left(Y_{0}\right)=\partial_{A} Y_{0}^{M} G_{M N} \partial_{B} Y_{0}^{N}$, they do not spoil the large- $D$ limit.

The equations of motion following from $(4.28)$ are

$$
\begin{gathered}
\sqrt{\operatorname{sdet} H_{C D}} H^{B A}=-i \lambda^{A B}, \\
H_{A B}-H_{A B}\left(Y_{0}\right)= \\
\alpha_{0} \frac{\delta}{\delta \lambda^{A B}} \operatorname{str}_{H} \ln \left(\frac{i}{\sqrt{\operatorname{sdet} H_{E F}}} \partial_{C} \lambda^{D C} \partial_{D}\right), \\
\partial_{A}\left(\lambda^{B A} \partial_{B} Y_{0}^{M}\right)=0 .
\end{gathered}
$$

To find a solution, consider first an infinite planar world-sheet in space-time. We can expect the classsical solution $\lambda^{A B}$ and $H_{A B}$ to be independent of $\sigma^{a}$. It might be tempting to assume their independence of $\theta^{\alpha}$ as well. This would lead to the following Ansatz:

$$
H_{A B}\left(\sigma^{a}, \theta^{\alpha}\right)=\omega H_{A B}\left(Y_{0}\right)=\omega H_{A B}^{(0)}
$$

with $\omega$ a $\left(\theta^{\alpha}\right.$-independent $)$ constant. Here $H_{A B}^{(0)}$ is the flat supermetric

$$
H_{a b}^{(0)}=\delta_{a b}, \quad H_{\alpha \beta}^{(0)} \equiv \epsilon_{\alpha \beta}, \quad H_{a \alpha}^{(0)}=H_{\alpha a}^{(0)}=0 .
$$

Plugging this Ansatz into the mean-field equations, we can see immediately that the assumption of $\theta^{\alpha}$-independence cannot be correct; this Ansatz gives no saddle point. This may have been guessed even before any calculation, by simply noting that the Ansatz 
does not contain any dimensionful parameters, which makes it insufficient for dimensional transmutation that we expect in the large $D$ expansion on the basis of asymptotic freedom.

The Ansatz must be modified, and precise details will depend on the boundary conditions imposed on the world-sheet. It is natural to consider

$$
H_{a b}=(\omega+(\mu+\tau) \theta \bar{\theta}) \delta_{a b}, \quad H_{\alpha \beta}=(\omega+(\mu-\tau) \theta \bar{\theta}) \epsilon_{\alpha \beta},
$$

with $\tau$ and $\mu$ parameters to be determined by the saddle-point condition (4.29). The dimensionful parameter $\tau$ plays the role of effective string tension,

$$
\int_{\Sigma} d^{2} \sigma d^{2} \theta \sqrt{\operatorname{sdet} H_{A B}}=\tau \int_{\Sigma} d^{2} \sigma d^{2} \theta \sqrt{h} \theta \bar{\theta}=\tau \int_{\Sigma} d^{2} \sigma \sqrt{h} .
$$

One can easily see that for non-zero $\tau$, the modified Ansatz (4.32) does lead to a saddle point, with the saddle-point value of $\tau$ determined via

$$
\tau \sim \Lambda^{2} \exp \left(-\frac{4 \pi}{D \alpha_{0}}\right)
$$

This is a standard result of dimensional transmutation in an asymptotically free theory.

Note that the dynamically generated tension $\tau$ breaks the Parisi-Sourlas supersymmetry on the world-sheet. Indeed, the presence of the $\theta$-dependent term in (4.32) means that the saddle-point value of $H_{A B}$ is not invariant under the scalar Parisi-Sourlas supersymmetries $Q$ and $\bar{Q}$ of (4.10). Unless the theory flows into a non-trivial infrared fixed point, we should expect that the long-distance behavior will be dominated by the tension term, dynamically generated by dimensional transmutation. The Parisi-Sourlas supersymmetry is only restored asymptotically in the ultraviolet, where it effectively plays the role of topological symmetry.

So far we have studied the large- $D$ theory on an infinite plane $\mathbf{R}^{2}$, and have argued that dimensional transmutation generates a non-zero string tension. In order to obtain any information about the string spectrum, one should study the theory on a world-sheet of topology $\mathbf{R}^{1} \times \mathbf{S}^{1}$, which correspond to the free closed string. Asymptotic freedom and dimensional transmutation imply that the ground state of the closed string is described by the full non-perturbative vacuum of the world-sheet field theory, on a world-sheet with non-trivial topology $\mathbf{R}^{1} \times \mathbf{S}^{1}$.

Alternatively, one can consider the following space-time point of view. In elementary closed string theory, where we have linear Regge trajectories, the ground state of the closed 
string is just the perturbative Fock vacuum, with at most a finite number of world-sheet oscillators excited. In the classical bosonic rigid string, the non-zero string rigidity prevents the classical ground state 5 from collapsing into a point, the ground state becomes a hoop of non-zero radius, and the Regge trajectories are consequently bent at low masses [22]. In our supersymmetric model, however, this does not happen classically on the world-sheet. Since there is no length scale in the classical world-sheet Lagrangian, there is nothing that would fix the radius of the hoop. Classically, there is no tension, and the string rigidity decompactifies the classical world-sheet. In the quantum theory, on the other hand, the length scale generated by dimensional transmutation gives a non-zero tension to the string, and the radius (of extrinsic curvature) of the string in its ground state is set dynamically.

One very important issue which is not entirely clear concerns unitarity of the theory. On the face of it, we seem to have two sources of possible violations of unitarity. The Lagrangian is of higher order in derivatives - a property shared by the bosonic rigid string, where it leads to ghost states at least in perturbation theory at small $\alpha_{0}$. In addition to that, our Parisi-Sourlas supersymmetric theory has fields with the "wrong" statistics, namely the fermions $\psi^{\mu}$ and $\chi^{\mu}$, which represent another possible source of ghost states. However, things may not really be as bad as they seem. Due to the local Parisi-Sourlas supersymmetry, gauge invariant states satisfy $Q \mid$ phys $\rangle=0$. This means that as as long as supersymmetry is unbroken, which is the case asymptotically in the ultraviolet, the theory is effectively a topological string theory, and unitarity should not be violated. We have argued that due to the asymptotic freedom of the theory, world-sheet supersymmetry is broken at all scales, and is only asymptotically restored in the UV limit. At long distances, the dynamically generated tension term dominates (assuming that the theory does not flow into a non-trivial IR fixed point), and the string is described by an effective NambuGoto Lagrangian. In this sense, the full supersymmetric rigid string theory provides an ultraviolet regularization of the Nambu-Goto string theory, in a way compatible with the apparent absence of conventional stringy degrees of freedom in the ultraviolet.

\subsection{String Tension in Two Dimensions and the Superpotential}

In two space-time dimensions, world-sheet supersymmetry stays unbroken at all scales, non-zero tension is not dynamically generated, and we need another mechanism for incor-

5 For the purpose of this argument, we can ignore the perturbative instability of the classical bosonic rigid string. 
porating non-zero tension in the theory. This can be done if we relax the global symmetry restrictions and permit soft breaking of the full space-time super-Poincaré symmetry $\operatorname{IOSp}(2 \mid 2)$ to its bosonic, Poincaré subgroup ISO(2).

Once we relax the condition of space-time $\operatorname{IOSp}(2 \mid 2)$ symmetry, we can write down a "superpotential" term

$$
\delta \mathcal{L}=\tau_{0} \int_{\Sigma} d^{2} \sigma d^{2} \theta \sqrt{\operatorname{sdet} H_{A B}} \Theta \bar{\Theta} .
$$

This term of course respects the local Parisi-Sourlas supersymmetry on the world-sheet. The coupling constant $\tau_{0}$ has canonical dimension two in mass units, and will play the role of the string tension as we are just about to see.

In order to make contact with the topological string formulation of Section 3.1b, we want to partially fix the gauge by choosing the fermionic Monge gauge (4.16) again. In this gauge, the remaining symmetries are those of the topological rigid string of Section 3.1, and the superpotential becomes

$$
\delta \mathcal{L}=\tau_{0} \int_{\Sigma} d^{2} \sigma \sqrt{h}\left(1+S_{0}+\ldots\right)
$$

Here $S_{0}$ is the $\theta=\bar{\theta}=0$ component of $S$. In terms of the component fields, $S_{0}$ is equal to the scalar product of the normal components of $\psi$ and $\chi$ :

$$
S_{0}=\psi \cdot \chi-\psi \cdot \partial_{a} x h^{a b} \partial_{b} x \cdot \chi
$$

Thus, $\tau_{0}$ behaves like the string tension, and the superpotential is equivalent to the NambuGoto Lagrangian, improved by a finite number of fermionic corrections.

This term precisely corresponds to the tension term in [12], eq. (6.21). It is interesting to notice that in the fully supersymmetric theory before gauge fixing, this term respects both local world-sheet supersymmetry and space-time Poincaré symmetry. After the partial gauge fixing, however, this term explicitly breaks the residual global fermionic symmetry that corresponds to the topological BRST charge in the formal topological interpretation of the theory. This explains why the non-zero tension case was somewhat difficult to understand in the conventional framework of two-dimensional topological rigid string theory [12]. Even in two space-time dimensions, it is natural to consider the more general framework of rigid string theory with local Parisi-Sourlas supersymmetry.

I benefited from useful discussions with Tom Banks, Mike Douglas, David Gross, Clifford Johnson, and Sasha Polyakov. I wish to thank the Aspen Center for Physics and the Rutgers High-Energy Theory Group for their generous hospitality and stimulating atmosphere 
during the initial and final stages of this work, respectively. This work has been supported by a Sherman Fairchild Prize Fellowship and by DOE grant DE-FG03-92-ER 40701. 


\section{References}

[1] J.M. Maldacena, "The Large N Limit of Superconformal Field Theories and Supergravity," hep-th/9711200.

[2] E. Witten, "Anti-de Sitter Space and Holography," hep-th/9802150.

[3] S.S. Gubser, I.R. Klebanov and A.M. Polyakov, "Gauge Theory Correlators from Non-Critical String Theory," Phys. Lett. B428 (1998) 105, hep-th/9802109.

[4] E. Witten, "Anti-de Sitter Space, Thermal Phase Transition, and Confinement in Gauge Theories," hep-th/9803131.

[5] J. Polchinski, "Strings and QCD?," hep-th/9211045.

[6] A.M. Polyakov, "String Theory and Quark Confinement," Nucl. Phys. Proc. Suppl. 68 (1998) 1, hep-th/9711002; "Confining Strings," hep-th/9607049.

[7] D.J. Gross and H. Ooguri, "Aspects of Large N Gauge Theory Dynamics as Seen by String Theory," hep-th/9805129.

[8] C. Csáki, H. Ooguri, Y. Oz and J. Terning, "Glueball Mass Spectrum from Supergravity," hep-th/9806021

H. Ooguri, H. Robins and J. Tannenhauser, "Glueballs and Their Kaluza-Klein Cousins," hep-th/9806171

R. de Mello Koch, A. Jevicki, M. Mihailescu and J. Nunez, "Evaluation of Glueball Masses from Supergravity," hep-th/9806125.

[9] M. Li, "t Hooft Vortices and Phases of Large N Gauge Theory," hep-th/9804175; "Evidence for Large N Phase Transition in $N=4$ Super Yang-Mills Theory at Finite Temperature," hep-th/9807196.

[10] P. Hořava, "Topological Strings and QCD in Two Dimensions," in: "Quantum Field Theory and String Theory," Proceedings of the Cargèse Workshop on "New Developments in String Theory, Conformal Models, and Topological Field Theory," May 1993; eds: L. Baulieu et al. (Plenum Press, 1995), hep-th/9311156.

[11] S. Cordes, G. Moore and S. Ramgoolam, "Large- $N$ 2D Yang-Mills Theory and Topological String Theory," Commun. Math. Phys. 185 (1997) 543, hep-th/9402107.

[12] P. Hořava, "Topological Rigid String Theory and Two-Dimensional QCD," Nucl. Phys. B463 (1996) 238, hep-th/9507060.

[13] L. Susskind and E. Witten, "The Holographic Bound in Anti-de Sitter Space," hepth/9805114.

[14] A.W. Peet and J. Polchinski, "UV/IR Relations in AdS Dynamics," hep-th/9809022.

[15] D.J. Gross, "Two Dimensional QCD as a String Theory," Nucl. Phys. B400 (1993) 161, hep-th/9212149

D.J. Gross and W. Taylor, "Two Dimensional QCD is a String Theory," Nucl. Phys. B400 (1993) 181, hep-th/9301068; "Twists and Wilson Loops in the String Theory of Two Dimensional QCD," Nucl. Phys. B403 (1993) 395, hep-th/9303046. 
[16] A. Migdal, "Recursion Equations in Gauge Field Theories," Zh. Eksp. Theor. Fiz. 69 (1975) 810

B.Ye. Rusakov, "Loop Averages and Partition Functions in $U(N)$ Gauge Theory in Two Dimensional Manifolds," Mod. Phys. Lett. A5 (1990) 693.

[17] E. Witten, "On Quantum Gauge Theories in Two Dimensions," Commun. Math. Phys. 141 (1991) 153; "Two Dimensional Yang-Mills Theory Revisited," J. Geom. Phys. 9 (1992) 303, hep-th/9204083.

[18] V. Mathai and D. Quillen, "Superconnections, Thom Classes, and Equivariant Differential Forms," Topology 25 (1986) 85

M.F. Atiyah and L. Jeffrey, "Topological Lagrangians and Cohomology," J. Geom. Phys. 7 (1990) 119.

[19] A.M. Polyakov, "Fine Structure of Strings," Nucl. Phys. B268 (1986) 406.

[20] H. Kleinert, "The Membrane Properties of Condensing Strings," Phys. Lett. B174 (1986) 335.

[21] A.M. Polyakov, "Gauge Fields and Strings" (Harwood Academic Publishers, 1987).

[22] R.D. Pisarski, "Soluble Theory with Massive Ghosts," Phys. Rev. D28 (1983) 2547; "Smooth Strings at Large Dimension," Phys. Rev. D38 (1988) 578; "Perturbative Stability of Smooth Strings," Phys. Rev. Lett. 58 (1987) 1300; "Heavy and Smooth Strings in QCD," in: "String Theory - Quantum Cosmology and Quantum Gravity; Integrable and Conformal Invariant Theories," eds: H.J. de Vega and N. Sánchez (World Scientific, 1987)

E. Braaten, R.D. Pisarski and S.-M. Tse, "Static Potential for Smooth Strings," Phys. Rev. Lett. 58 (1987) 93.

[23] C. Itoi and H. Kubota, "BRST Quantization of the String Model with Extrinsic Curvature," Phys. Lett. B202 (1988) 381; "Gauge Invariance Based on the Extrinsic Geometry in the Rigid String," Z. Phys. C44 (1989) 337.

[24] P. Olesen and S.-K. Yang, "Static Potential in a String Model with Extrinsic Curvatures," Nucl. Phys. B283 (1987) 73.

[25] F. Alonso and D. Espriu, "On the Fine Structure of Strings," Nucl. Phys. B283 (1987) 393.

[26] J. Polchinski and Z. Yang, "High Temperature Partition Function of the Rigid String," hep-th/9205043.

[27] S.-J. Rey and J. Yee, "Macroscopic Strings as Heavy Quarks of Large N Gauge Theory and Anti-de Sitter Supergravity," hep-th/9803001

J.M. Maldacena, "Wilson Loops in Lage N Field Theories," Phys. Rev. Lett. 80 (1998) 4859, hep-th/9803002.

[28] A.M. Polyakov, "The Wall of the Cave," hep-th/9809057. 
[29] G. Parisi and N. Sourlas, "Random Magnetic Fields, Supersymmetry, and Negative Dimensions," Phys. Rev. Lett. 43 (1979) 744; "Self Avoiding Walk and Supersymmetry," J. Physique Lett. 41 (1980) L-406; "Critical Behavior of Branched Polymers and the Lee-Yang Edge Singularity," Phys. Rev. Lett. 46 (1981) 871.

[30] A.J. McKane, "Reformulation of $n \rightarrow 0$ Models Using Anticommuting Scalar Fields," Phys. Lett. A76 (1980) 22.

[31] N. Sourlas, "Introduction to Supersymmetry in Condensed Matter Physics," Physica 15D (1985) 115

J.L. Cardy, "Nonperturbative Aspects of Supersymmetry in Statistical Mechanics," Physica 15D (1985) 123

Y. Shapir, "Supersymmetric Statistical Models on the Lattice," Physica 15D (1985) 129.

[32] G. Parisi and Y.-S. Wu, "Perturbation Theory without Gauge Fixing," Sci. Sinica 24 (1981) 483

G. Parisi and N. Sourlas, "Supersymmetric Field Theories and Stochastic Differential Equations," Nucl. Phys. B206 (1982) 321.

[33] B. McClain, A. Niemi, C. Taylor and L.C.R. Wijewardhana, "Superspace, Dimensional Reduction, and Stochastic Quantization," Nucl. Phys. B217 (1983) 430.

[34] M. Namiki, "Stochastic Quantization," L. N. Phys. Monographs m9 (Springer Verlag, 1992).

[35] J. Greensite and M.B. Halpern, "Quenched Master Fields," Nucl. Phys. B211 (1983) 343

M.R. Douglas, "Stochastic Master Fields," Phys. Lett. B344 (1995) 117, hepth/9411025.

[36] P.G. de Gennes, "Exponents for the Excluded Volume Problem as Derived by the Wilson Method," Phys. Lett. A38 (1972) 339

J. des Cloizeaux, "Lagrangian Theory for a Self-Avoiding Random Chain," Phys. Rev. A10 (1974) 1665

J. des Cloizeaux, "The Lagrangian Theory of Polymer Solutions at Intermediate Concentrations," J. Physique 36 (1975) 281.

[37] T.C. Lubensky and J. Isaacson, "Statistics of Lattice Animals and Dilute Branched Polymers," Phys. Rev. A20 (1979) 2130.

[38] J. des Cloizeaux and G. Jannink, "Les Polymères en Solution: Leur Modélisation et leur Structure" (Les Editions de Physique, 1987); English translation: "Polymers in Solution: Their Modelling and Structure" (Oxford University Press, 1990).

[39] I. Pesando and D. Cassi, "Polymers and Topological Field Theory," Mod. Phys. Lett. B6 (1992) 485.

[40] P. Nelson and T. Powers, "Renormalization of Chiral Couplings in Tilted Bilayer Membranes," J. Phys. II France 3 (1993) 1535, cond-mat/9305014. 
[41] W. Cai, T.C. Lubensky, P. Nelson and T. Powers, "Measure Factors, Tension, and Correlations of Fluid Membranes," J. Phys. II France 4 (1994) 931, cond-mat/9401020.

[42] "Statistical Mechanics of Membranes and Surfaces," Proceedings of the Jerusalem Winter School of Theoretical Physics, Vol. 5, eds: D. Nelson, T. Piran and S. Weinberg (World Scientific, 1988).

[43] F. David, "Geometry and Field Theory of Random Surfaces and Membranes," in [42], p. 157

F. David and S. Leibler, "Vanishing Tension of Fluctuating Membranes," J. Phys. II France 1 (1991) 959. 